\title{
Lorentz violation in brane cosmology, accelerated expansion and fundamental constants
}

\author{
F. Ahmadi* S. Jalalzadeh ${ }^{\dagger}$ and H. R. Sepangi ${ }^{\ddagger}$ \\ Department of Physics, Shahid Beheshti University, Evin, Tehran 19839, Iran
}

October 15, 2018

\begin{abstract}
The notion of Lorentz violation in four dimensions is extended to a 5 -dimensional brane-world scenario by utilizing a dynamical vector field assumed to point in the bulk direction, with Lorentz invariance holding on the brane. The cosmological consequences of this theory consisting of the time variation in the gravitational coupling $G$ and cosmological term $\Lambda_{4}$ are explored. The brane evolution is addressed by studying the generalized Friedmann and Raychaudhuri equations. The behavior of the expansion scale factor is then considered for different possible scenarios where the bulk cosmological constant is zero, positive or negative.
\end{abstract}

\section{Introduction}

The possibility that Lorentz invariance may be violated at high energies in $4 D$ with testable consequence has become a subject of much interest in the past few years [1]. Tentative results from quantum gravity and string theory point to a ground state that may not be Lorentz invariant [2]. String theory also predicts that we may live in a universe with non-commutative coordinates [3] leading to violation of Lorentz invariance [4]. In addition, astrophysical observations point to the presence of high energy cosmic rays about the Greisen-Zatsepin-Kuzmin cutoff [5], results which may be explained by a breaking down of Lorentz invariance [6]-[14]. Moreover, due to the unboundedness of the boost parameter, exact Lorentz invariance, while mathematically elegant, is unverifiable and therefore suspect. Most works exploring possible Lorentz violation have focused on non gravitational physics, i.e. flat space-times. Much less has been done to investigate Lorentz breaking in curved space-times. In flat space-times, Lorentz violation is described by couplings to constant symmetry breaking tensors $V_{a}, W_{a b}$ and so on. To formulate Lorentz breaking in a curved space-time without destroying general covariance such tensors must become dynamical tensor fields that satisfy effective field equations.

A straightforward method of implementing local Lorentz violation in a gravitational setting is to imagine the existence of a tensor field with a non-vanishing expectation value and couple this tensor to gravity or matter fields. The simplest example of this approach is to consider a single time-like vector field with fixed norm. A special case of this theory was first introduced as a mechanism for Lorentz violation by Kostelecky and Samuel [15]. In a different context, Bekenstein has proposed a theory of gravity with a fixed-norm vector in order to mimic the effects of dark matter [16]. Also, studies of vector fields in a cosmological setting without the fixed norm have been done in [17]-[20]. This vector field picks out a preferred frame at each point in space-time and any matter field coupled to it will experience a violation of local Lorentz invariance [21, 22].

*email: fa-ahmadi@sbu.ac.ir

†email: s-jalalzadeh@sbu.ac.ir

${ }^{\ddagger}$ email: hr-sepangi@sbu.ac.ir 
General relativity cannot describe gravity at high enough energies and must be replaced by quantum gravity theory. The physics responsible for making a sensible quantum theory of gravity is revealed only at the Planck scale. This cut-off scale marks the point where our old description of nature breaks down and it is not inconceivable that one of the victims of this break down is Lorentz invariance. It is thus interesting to test the robustness of this symmetry at the highest energy scales $[13,23,24]$. As usual in high energy physics, if the scale characterizing new physics is too high then it cannot be reached directly in collider experiments. In this case cosmology is the only place where the effects of new physics can be indirectly observed. Brane-world models offer a phenomenological way to test some of the novel predictions and corrections to general relativity that are implied by M-theory. Lorentz violating effects have also been studied within the context of the brane-world scenarios. In such models, the space-time globally violates $4 D$ Lorentz invariance, leading to apparent violations of Lorentz invariance from the brane observer's point of view due to bulk gravity effects. These effects are restricted to the gravity sector of the effective theory while the well measured Lorentz invariance of particle physics remains unaffected in these scenarios [25, 26]. In a similar vein, Lorentz invariance violation has been employed to shed some light on the possibility of signals travelling along the extra dimension outside our visible universe [27]. In a different approach a brane-world toy model has been introduced [28] in an inflating $5 D$ brane-world setup with violation of $4 D$ Lorentz invariance at an energy scale $k$.

In this paper we consider Lorentz violation in a brane-world scenario where it occurs in the bulk space along the extra dimension. This is complementary to the above mentioned brane world scenarios in that the Lorentz violation affects the bulk space rather than the $4 D$ brane. To this end, we consider the theory suggested by Jacobson and Mattingly [29,30] where the gravitational effects of the vector fields are described in four dimensions. We generalize the theory to include higher dimensional gravity and in particular the brane-world scenarios. As is well known, brane-worlds are often studied within the framework of the $5 D$ Einstein field equations projected onto the $4 D$ brane, a prime example of which is the formulation of Shiromizu, Maeda and Sasaki (SMS) [31]. To study Lorentz violation, we consider a vector $N^{A}$ along the extra dimension. In doing so a local frame at a point in space-time is inevitably selected as the preferred frame. Put in other words, the existence of the brane defines a preferred direction in the bulk. We then move on to study the effects of local Lorentz violation on the dynamics of the brane. We assume no coupling between the matter fields and the vector field as the brane observer does not feel the presence of the preferred frame. This additional field modifies the $4 D$ Einstein equations with cosmological implications which we investigate by studying the resulting Friedmann and Raychaudhuri equations on the brane. We find that Lorentz violation allows for the construction of models which show variations in the fundamental physical "constants." Finally, we determine the brane evolution for different possible scenarios where the bulk cosmological constant is negative, zero or positive.

\section{Field Equations}

In the usual brane-world scenarios the space-time is identified with a singular hypersurface (or 3brane) embedded in a five-dimensional bulk. Suppose now that $N^{A}$ is a given vector field along the extra dimension, effectively making the associated frame a preferred one. The theory we consider consists of the vector field $N^{A}$ minimally coupled to gravity with an action of the form ${ }^{1}$.

$$
S=\int d^{5} x \sqrt{-{ }^{(5)} g}\left[\frac{1}{2 k_{5}^{2}}\left({ }^{(5)} R+\mathcal{L}_{N}\right)+\mathcal{L}_{m}\right]
$$

where $k_{5}^{2}$ is a constant introduced for dimensional considerations, $\mathcal{L}_{N}$ is the vector field Lagrangian density while $\mathcal{L}_{m}$ denotes the Lagrangian density for all the other matter fields. In order to preserve general covariance, $N^{A}$ is taken to be a dynamical field. The Lagrangian density for the vector field

\footnotetext{
${ }^{1}$ The upper case Latin indices take the values $0,1,2,3$ and 5 while the Greek indices run from 0 to 3.
} 
is written as

$$
\mathcal{L}_{N}=K^{A B}{ }_{C D} \nabla_{A} N^{C} \nabla_{B} N^{D}+\lambda\left(N^{A} N_{A}-\epsilon\right)
$$

where

$$
K_{C D}^{A B}=-\beta_{1} g^{A B} g_{C D}-\beta_{2} \delta_{C}^{A} \delta_{D}^{B}-\beta_{3} \delta_{D}^{A} \delta_{C}^{B}
$$

Here, $\beta_{i}$ are dimensionless parameters, $\epsilon=-1$ or $\epsilon=1$ depending on whether the extra dimension is space-like or time-like respectively and $\lambda$ is a Lagrange multiplier. This is a slight simplification of the theory introduced by Jacobson and Mattingly [29], where we have neglected a quartic self-interaction term of the form $\left(N^{A} \nabla_{A} N^{B}\right)\left(N^{c} \nabla_{c} N_{B}\right)$ as has been done in [32]. For their theory to be well-defined, the following conditions should hold

$$
\beta_{1}>0, \quad \frac{\left(\beta_{1}+\beta_{2}+\beta_{3}\right)}{\beta_{1}} \geq 0, \quad \frac{\left(\beta_{1}+\beta_{2}+\beta_{3}\right)}{\beta_{1}} \leq 1, \quad \beta_{1}+\beta_{3} \leq 0 .
$$

The first of these arises from the need for a positive-definite Hamiltonian for the perturbation, the next two from demanding non-tachyonic and subluminal propagation of the spin-0 degrees of freedom respectively, and the last from insisting that gravity waves propagate subluminally $[32,33]$. In the present work however, we will not be relying on these constraints for our analysis. We also define a current tensor $J^{A}$ via

$$
J^{A}{ }_{C} \equiv K^{A B}{ }_{C D} \nabla_{B} N^{D}
$$

Note that the symmetry of $K^{A B}{ }_{C D}$ means that $J^{B}{ }_{D}=K^{A B}{ }_{C D} \nabla_{A} N^{C}$. With these definitions the equation of motion obtained by varying the action with respect to $N^{A}$ is

$$
\nabla_{A} J^{A B}=\lambda N^{B} .
$$

The equation of motion for $\lambda$ enforces the fixed norm constraint

$$
g_{A B} N^{A} N^{B}=\epsilon, \quad \epsilon^{2}=1 .
$$

Choosing $\epsilon=1$ ensures that the vector will be time-like. Multiplying both sides of (5) by $N_{B}$ and using (7), we find

$$
\lambda=\epsilon N_{B} \nabla_{A} J^{A B} .
$$

One may also project into a subspace orthogonal to $N^{A}$ by acting the projection tensor $P^{C}{ }_{B}=$ $-\epsilon N^{C} N_{B}+\delta_{B}^{C}$ on equation (6) to obtain

$$
\nabla_{A} J^{A C}-\epsilon N^{C} N_{B} \nabla_{A} J^{A B}=0 .
$$

This equation determines the dynamics of $N^{A}$, subject to the fixed-norm constraint.

In taking the variation, it is important to distinguish the variables that are independent. Our dynamical degrees of freedom are the inverse metric $g^{A B}$ and the contravariant vector field $N^{A}$. Hence, the Einstein equations in the presence of both the matter and vector fields in bulk space are $[34]$

$$
{ }^{(5)} G_{A B}={ }^{(5)} R_{A B}-\frac{1}{2} g_{A B}{ }^{(5)} R=k_{5}^{2(5)} T_{A B},
$$

where

$$
{ }^{(5)} T_{A B}={ }^{(5)} T_{A B}^{(m)}+\frac{1}{k_{5}^{2}} T_{A B}^{(N)} \cdot
$$

Here, $T_{A B}^{(m)}$ is the five-dimensional energy-momentum tensor and the stress-energy $T_{A B}^{(N)}$ is considered to have the following form $[32,35]$

$$
\begin{aligned}
T_{A B}^{(N)} & =2 \beta_{1}\left(\nabla_{A} N^{C} \nabla_{B} N_{C}-\nabla^{C} N_{A} \nabla_{C} N_{B}\right)-2\left[\nabla_{C}\left(N_{(A} J^{C}{ }_{B)}\right)+\nabla_{C}\left(N^{C} J_{(A B)}\right)\right. \\
& \left.-\nabla_{C}\left(N_{(A} J_{B)}{ }^{C}\right)\right]+2 \epsilon N_{D} \nabla_{C} J^{C D} N_{A} N_{B}+g_{A B} \mathcal{L}_{N} .
\end{aligned}
$$


Let us take the metric of the bulk space as

$$
d S^{2}=g_{\mu \nu}\left(x^{\alpha}, y\right) d x^{\mu} d x^{\nu}+\epsilon \phi^{2}\left(x^{\alpha}, y\right) d y^{2},
$$

where $\phi$ is a scalar field and we have used signature $(+---\epsilon)$ everywhere. The Einstein equations (10) contain the first and second derivatives of the metric with respect to the extra coordinate. These can be expressed in terms of geometrical tensors in $4 D$. In the absence of off-diagonal terms $\left(g_{5 \mu}=0\right)$ the dimensional reduction of the five-dimensional equations is particularly simple [36], [37]. The usual assumption is that our space-time is orthogonal to the extra dimension. Thus using equations (7) and (13), we can introduce the normal unit vector $N^{A}$ which is orthogonal to the hypersurfaces $y=$ const. as

$$
N^{A}=\frac{\delta_{5}^{A}}{\phi}, \quad N_{A}=(0,0,0,0, \epsilon \phi)
$$

The first partial derivatives can be written in terms of the extrinsic curvature

$$
K_{\mu \nu}=\frac{1}{2} \mathcal{L}_{N} g_{\mu \nu}=\frac{1}{2 \phi} \frac{\partial g_{\mu \nu}}{\partial y}, \quad K_{A 5}=0 .
$$

The second derivatives can be expressed in terms of the projection ${ }^{(5)} C_{\mu 5 \nu 5}$ of the bulk Weyl tensor to $5 D$

$$
{ }^{(5)} C_{A B C D}={ }^{(5)} R_{A B C D}-\frac{2}{3}\left({ }^{(5)} R_{A[C} g_{D] B}-{ }^{(5)} R_{B[C} g_{D] A}\right)+\frac{1}{6}\left({ }^{(5)} R g_{A[C} g_{D] B}\right) .
$$

The field equations (10) can be split up into three parts. Using the Gauss-Codazzi equations, the effective field equations in $4 D$ are

$$
\begin{aligned}
{ }^{(4)} G_{\mu \nu} & =\frac{2}{3} k_{5}^{2}\left[{ }^{(5)} T_{\mu \nu}+\left({ }^{(5)} T_{5}^{5}-\frac{1}{4}\left({ }^{(5)} T\right)\right) g_{\mu \nu}\right] \\
& -\epsilon\left(K_{\mu \alpha} K_{\nu}^{\alpha}-K K_{\mu \nu}\right)+\frac{\epsilon}{2} g_{\mu \nu}\left(K_{\alpha \beta} K^{\alpha \beta}-K^{2}\right)-\epsilon E_{\mu \nu}
\end{aligned}
$$

where

$$
E_{\mu \nu}={ }^{(5)} C_{\mu A \nu B} N^{A} N^{B}=-\frac{1}{\phi} \frac{\partial K_{\mu \nu}}{\partial y}+K_{\mu \gamma} K_{\nu}^{\gamma}-\epsilon \frac{\phi_{\mu ; \nu}}{\phi}-\epsilon \frac{k_{5}^{2}}{3}\left[{ }^{(5)} T_{\mu \nu}+\left({ }^{(5)} T_{5}^{5}-\frac{1}{2}\left({ }^{(5)} T\right)\right) g_{\mu \nu}\right] .
$$

Since the electric part of the Weyl tensor $E_{\mu \nu}$ is traceless, the requirement $E_{\mu}^{\mu}=0$ gives the inhomogeneous wave equation for $\phi$

$$
\phi_{; \mu}^{\mu}=-\epsilon \frac{\partial K}{\partial y}-\phi\left(\epsilon K_{\alpha \beta} K^{\alpha \beta}+{ }^{(5)} R_{5}^{5}\right)
$$

which is equivalent to ${ }^{(5)} G_{55}=k_{5}^{2(5)} T_{55}$ from (10). The remaining four equations are

$$
D_{\mu}\left(K_{\nu}^{\mu}-\delta_{\nu}^{\mu} K\right)=k_{(5)}^{2} \frac{{ }^{(5)} T_{5 \nu}}{\phi} .
$$

In the above expressions, the covariant derivatives are calculated with respect to $g_{\mu \nu}$, i.e., $D g_{\mu \nu}=0$.

\section{Brane-world considerations}

With an eye on the brane-world scenario, it is assumed that the five-dimensional energy-momentum tensor has the form

$$
{ }^{(5)} T_{A B}^{(m)}=\Lambda_{5} g_{A B}
$$


where $\Lambda_{5}$ is the cosmological constant in the bulk. Now, using equation (12) we may calculate ${ }^{(5)} T_{\mu \nu}$, ${ }^{(5)} T_{5}^{5}$ and ${ }^{(5)} T$, obtaining

$$
\begin{aligned}
{ }^{(5)} T_{\mu \nu} & =\frac{1}{k_{5}^{2}}\left[-4\left(\beta_{1}+\beta_{3}\right) K_{\mu \gamma} K_{\nu}^{\gamma}+2\left(\beta_{1}+\beta_{3}\right) K K_{\mu \nu}+\beta_{2} g_{\mu \nu} K^{2}+\frac{2\left(\beta_{1}+\beta_{3}\right)}{\phi} K_{\mu \nu, 5}\right. \\
& \left.+\frac{2 \beta 2}{\phi} g_{\mu \nu} K_{, 5}-\left(\beta_{1}+\beta_{3}\right) g_{\mu \nu} K_{\alpha \beta} K^{\alpha \beta}+2 \epsilon \beta_{1} \frac{\phi_{, \mu} \phi_{, \nu}}{\phi^{2}}-\epsilon \beta_{1} g_{\mu \nu} \frac{\phi_{, \alpha} \phi_{,}^{\alpha}}{\phi^{2}}\right]+\Lambda_{5} g_{\mu \nu}, \\
{ }^{(5)} T_{5}^{5} & =\frac{1}{k_{5}^{2}}\left[\left(\beta_{1}+\beta_{3}\right) K_{\alpha \beta} K^{\alpha \beta}+\beta_{2} K^{2}+2 \epsilon \beta_{1} g^{\mu \nu} \frac{\phi_{; \nu \mu}}{\phi}-\epsilon \beta_{1} \frac{\phi_{, \alpha} \phi_{,}^{\alpha}}{\phi^{2}}\right]+\Lambda_{5}, \\
{ }^{(5)} T & =\frac{1}{k_{5}^{2}}\left[-3\left(\beta_{1}+\beta_{3}\right) K_{\alpha \beta} K^{\alpha \beta}+2\left(\beta_{1}+\beta_{3}\right) K^{2}+5 \beta_{2} K^{2}+\frac{2}{\phi}\left(\beta_{1}+\beta_{3}\right) K_{, 5}+\frac{8}{\phi} \beta_{2} K_{, 5}\right. \\
& \left.-3 \epsilon \beta_{1} \frac{\phi_{, \alpha} \phi_{,}^{\alpha}}{\phi^{2}}+2 \epsilon \beta_{1} g^{\mu \nu} \frac{\phi_{; \mu \nu}}{\phi}\right]+5 \Lambda_{5} .
\end{aligned}
$$

Let us now substitute equations (22), (23) and (24) in equation (17) and use the following equations

$$
\begin{aligned}
& \frac{1}{\phi}\left(K_{\mu \nu, 5}-\frac{1}{4} g_{\mu \nu} K_{, 5}\right)=-\frac{1}{2}\left({ }^{(4)} R_{\mu \nu}-\frac{1}{4} g_{\mu \nu}^{(4)} R\right)-\left(\frac{\phi_{; \nu \mu}}{\phi}-\frac{1}{4} g_{\mu \nu} \frac{\phi_{; \alpha}^{\alpha}}{\phi}\right) \\
& +\frac{1}{2}\left(K_{\mu}^{\alpha} K_{\alpha \nu}+K K_{\mu \nu}\right)+\frac{3}{8} g_{\mu \nu}\left(K_{\alpha \beta} K^{\alpha \beta}-\frac{1}{3} K^{2}\right)-\frac{3}{2} E_{\mu \nu}, \\
& { }^{(4)} R=\epsilon K^{2}-\epsilon K_{\alpha \beta} K^{\alpha \beta}-2\left(R_{5}^{5}-\frac{1}{2} g_{5}^{5} R\right)=\epsilon K^{2}-\epsilon K_{\alpha \beta} K^{\alpha \beta}-2 k_{5}^{2} T_{5}^{5}=\epsilon K^{2} \\
& \text { - } \epsilon K_{\alpha \beta} K^{\alpha \beta}-2 k_{5}^{2} \Lambda_{5}-2\left(\beta_{1}+\beta_{3}\right) K_{\alpha \beta} K^{\alpha \beta}-2 \beta_{2} K^{2}-4 \epsilon \beta_{1} g^{\alpha \beta} \frac{\phi_{; \alpha \beta}}{\phi}+2 \epsilon \beta_{1} \frac{\phi_{, \alpha} \phi_{,}^{\alpha}}{\phi^{2}},
\end{aligned}
$$

where (25) may be obtained from an equation for the electric part of the Weyl tensor [31] and (26) can be derived from the Gauss equation. Now, upon defining the following new set of parameters

$$
\begin{aligned}
& \alpha_{1}=2\left(\beta_{1}+\beta_{3}\right), \quad \alpha_{2}=\frac{2 \epsilon\left(\beta_{1}+\beta_{2}+\beta_{3}\right)}{3-2 \epsilon\left(\beta_{1}+4 \beta_{2}+\beta_{3}\right)}, \\
& \alpha_{3}=\frac{\alpha_{1}\left(3+\epsilon-2 \beta_{2}\right)}{6}-\beta_{2}, \quad \alpha_{4}=\frac{\alpha_{1}\left(6+\epsilon+\alpha_{1}\right)}{6}, \quad \alpha_{5}=\epsilon \beta_{1},
\end{aligned}
$$

equation (17) becomes

$$
\begin{aligned}
{ }^{(4)} G_{\mu \nu} & =\frac{k_{5}^{2}}{2} g_{\mu \nu} \Lambda_{5}-\frac{3\left(\epsilon+\alpha_{1}\right)}{\left(3+\alpha_{1}\right)}\left(K_{\mu \gamma} K_{\nu}^{\gamma}-K K_{\mu \nu}\right)-\frac{3\left(\epsilon+\alpha_{3}\right)}{2\left(3+\alpha_{1}\right)} g_{\mu \nu} K^{2} \\
& +\frac{3\left(\epsilon+\alpha_{4}\right)}{2\left(3+\alpha_{1}\right)} g_{\mu \nu} K_{\alpha \beta} K^{\alpha \beta}+\left[\frac{\alpha_{1}\left(\alpha_{5}+\frac{1}{2}\right)+3 \alpha_{5}}{\left(3+\alpha_{1}\right)}\right] g_{\mu \nu} \frac{\phi_{; \alpha}^{\alpha}}{\phi}-\frac{\alpha_{5}\left(5+\alpha_{1}\right)}{2\left(3+\alpha_{1}\right)} g_{\mu \nu} \frac{\phi_{, \alpha} \phi_{,}^{\alpha}}{\phi^{2}} \\
& -\frac{2 \alpha_{1}}{\left(3+\alpha_{1}\right)} \frac{\phi_{; \nu \mu}}{\phi}+\left[\frac{4 \alpha_{5}}{\left(3+\alpha_{1}\right)}\right] \frac{\phi_{, \mu} \phi_{, \nu}}{\phi^{2}}-\frac{3\left(\epsilon+\alpha_{1}\right)}{\left(3+\alpha_{1}\right)} E_{\mu \nu} .
\end{aligned}
$$

Note that $\left(3+\alpha_{1}\right)$ is the coefficient of the four-dimensional Einstein tensor. It therefore provides a relation among the extrinsic curvature, the electric part of the Weyl tensor and scalar field $\phi$ when $\alpha_{1}=-3$. We take $\alpha_{1} \neq-3$ from hereon. In the spirit of the brane world scenario, we assume $Z_{2}$ symmetry about our brane, considered to be a hypersurface $\Sigma$ at $y=0$, and write the five-dimensional energy-momentum tensor ${ }^{(5)} T_{A B}^{(m)}$ in the resulting $Z_{2}$-symmetric brane universe as

$$
{ }^{(5)} T_{A B}^{(m)}=\Lambda_{5} g_{A B}+{ }^{(5)} T_{A B}^{(\text {brane })},
$$


where ${ }^{(5)} T_{A B}^{(\text {brane })}$ is the energy-momentum tensor of the matter on the brane with ${ }^{(5)} T_{A B}^{(b r a n e)} N^{A}=0$ and

$$
{ }^{(5)} T_{A B}^{(\text {brane })}=\delta_{A}^{\mu} \delta_{B}^{\nu} \tau_{\mu \nu} \frac{\delta(y)}{\phi} .
$$

In order to obtain the Einstein equations on the brane, we need to find an expression for the extrinsic curvature of $\Sigma$. For this, we use equation (10) and find

$$
\begin{aligned}
{ }^{(5)} R_{\mu \nu} & =k_{5}^{2}\left[\frac{-2}{3} \Lambda_{5} g_{\mu \nu}+\frac{\delta(y)}{\phi}\left(\tau_{\mu \nu}-\frac{1}{3} g_{\mu \nu} \tau\right)\right]-4\left(\beta_{1}+\beta_{3}\right) K_{\mu \gamma} K_{\nu}^{\gamma}+2\left(\beta_{1}+\beta_{3}\right) K K_{\mu \nu} \\
& -\frac{2}{3}\left(\beta_{1}+\beta_{2}+\beta_{3}\right) g_{\mu \nu} K^{2}+\frac{2\left(\beta_{1}+\beta_{3}\right)}{\phi} K_{\mu \nu, 5}-\frac{2}{3}\left(\beta_{1}+\beta_{2}+\beta_{3}\right) g_{\mu \nu} \frac{K_{, 5}}{\phi} \\
& +2 \epsilon \beta_{1} \frac{\phi_{, \mu} \phi_{, \nu}}{\phi^{2}}-\frac{2}{3} \epsilon \beta_{1} g_{\mu \nu} \frac{\phi_{; \alpha}^{\alpha}}{\phi} .
\end{aligned}
$$

On the other hand, for metric (13) we have

$$
{ }^{(5)} R_{\mu \nu}=-\epsilon \frac{\partial}{\partial y}\left(\frac{K_{\mu \nu}}{\phi}\right)+V_{\mu \nu}
$$

where

$$
V_{\mu \nu}{ }^{(4)} R_{\mu \nu}+\epsilon\left(2 K_{\mu \gamma} K_{\nu}^{\gamma}-K_{\mu \nu} K\right)-\frac{{ }^{(5)} \nabla_{\nu} \phi_{; \mu}}{\phi},
$$

with ${ }^{(5)} \nabla_{\nu} \phi_{\mu}=\phi_{\mu, \nu}-\Gamma_{\mu \nu}^{A} \phi_{A}$. We now substitute this into equation (31) and integrate across the brane noting that the metric is continuous. Although the derivatives $\partial g_{\mu \nu} / \partial y$ and $\partial \phi / \partial y$ are discontinuous across $\Sigma: y=0$, we make the usual physical assumption that they remain finite. Thus, $\lim _{\xi \rightarrow 0} \int_{-\xi / 2}^{\xi / 2} V_{\mu \nu} d y=0$ and using $Z_{2}$ symmetry and the set of parameters (28), we obtain

$$
\left.K_{\mu \nu}\right|_{\Sigma^{+}}=-\left.K_{\mu \nu}\right|_{\Sigma^{-}}=-\frac{\epsilon k_{5}^{2}}{2\left(1+\epsilon \alpha_{1}\right)}\left[\tau_{\mu \nu}-\frac{1}{3} g_{\mu \nu}\left(1+\alpha_{2}\right) \tau\right]
$$

To avoid unreal singularities in equations (28) and (34), it would be convenient to take $\alpha_{1}<-3$. Now, from equation (20) it follows that

$$
\left(\tau_{\nu}^{\mu}\right)_{; \mu}=-2\left(\epsilon+\alpha_{1}\right) \frac{(5) T_{5 \nu}}{\phi}-\alpha_{2} \tau_{, \nu}
$$

where

$$
{ }^{(5)} T_{5 \nu}=\frac{2 \epsilon}{k_{5}^{2}}\left(2 \beta_{1}+\beta_{2}+2 \beta_{3}\right) K_{\nu}^{\mu} \phi_{, \mu}+\frac{2 \epsilon}{k_{5}^{2}}\left(2 \beta_{2}-\beta_{1}\right) K \phi_{, \nu}-\frac{\epsilon \beta_{1}}{k_{5}^{2}} \frac{\phi_{, \nu 5}}{\phi} .
$$

Thus, the energy-momentum tensor $\tau_{\mu \nu}$ is not conserved on the brane and represents the total vacuum plus matter energy-momentum. It is usually separated in two parts,

$$
\tau_{\mu \nu}=\sigma g_{\mu \nu}+T_{\mu \nu}
$$

where $\sigma$ is the tension of the brane in $5 D$, which is interpreted as the vacuum energy of the brane world and $T_{\mu \nu}$ represents the energy-momentum tensor of ordinary matter in $4 D$. Using equations (34) and (37) and defining the following set of parameters

$$
\begin{aligned}
& \alpha_{6}=\frac{\alpha_{1}\left(1-2 \alpha_{2}\right)-2 \epsilon \alpha_{2}}{3} \\
& \alpha_{7}=\frac{\left(\epsilon+\alpha_{1}\right)\left(\alpha_{2}+\alpha_{2}^{2}\right)}{3}+\frac{\left(\alpha_{4}-3 \epsilon-4 \alpha_{3}\right)\left(\alpha_{2}+2 \alpha_{2}^{2}\right)}{9}-\frac{\left(\alpha_{3}+2 \alpha_{4}\right)}{18},
\end{aligned}
$$


we obtain the Einstein field equations with an effective energy-momentum tensor in $4 D$ as

$$
\begin{aligned}
{ }^{(4)} G_{\mu \nu} & =\Lambda_{4} g_{\mu \nu}+8 \pi G T_{\mu \nu}+k_{5}^{4} \Pi_{\mu \nu}-\frac{3\left(\epsilon+\alpha_{1}\right)}{\left(3+\alpha_{1}\right)} E_{\mu \nu}+\left[\frac{\alpha_{1}\left(\alpha_{5}+\frac{1}{2}\right)+3 \alpha_{5}}{\left(3+\alpha_{1}\right)}\right] g_{\mu \nu} \frac{\phi_{; \alpha}^{\alpha}}{\phi} \\
& -\frac{\alpha_{5}\left(5+\alpha_{1}\right)}{2\left(3+\alpha_{1}\right)} g_{\mu \nu} \frac{\phi_{, \alpha} \phi_{,}^{\alpha}}{\phi^{2}}-\frac{2 \alpha_{1}}{\left(3+\alpha_{1}\right)} \frac{\phi_{; \nu \mu}}{\phi}+\left[\frac{4 \alpha_{5}}{\left(3+\alpha_{1}\right)}\right] \frac{\phi_{, \mu} \phi_{, \nu}}{\phi^{2}},
\end{aligned}
$$

where

$$
\begin{gathered}
\Lambda_{4}=\frac{k_{5}^{2}}{2} \Lambda_{5}+\left[\frac{-\epsilon k_{5}^{4}+3 k_{5}^{4}\left(-\alpha_{1}+4 \alpha_{6}+16 \alpha_{7}+2 \alpha_{4}\right)}{4\left(3+\alpha_{1}\right)\left(1+\epsilon \alpha_{1}\right)^{2}}\right] \sigma^{2}, \\
8 \pi G=\left[\frac{-2 \epsilon k_{5}^{4}+3 k_{5}^{4}\left(-2 \alpha_{1}+4 \alpha_{6}\right)}{4\left(3+\alpha_{1}\right)\left(1+\epsilon \alpha_{1}\right)^{2}}\right] \sigma,
\end{gathered}
$$

and

$$
\begin{aligned}
\Pi_{\mu \nu} & =\frac{3}{4\left(3+\alpha_{1}\right)\left(1+\epsilon \alpha_{1}\right)^{2}}\left[-\left(\epsilon+\alpha_{1}\right) T_{\mu \gamma} T_{\nu}^{\gamma}+\left(\frac{\epsilon}{3}+\alpha_{6}\right) T T_{\mu \nu}\right. \\
& \left.-\left(\frac{\epsilon}{6}-\alpha_{7}\right) g_{\mu \nu} T^{2}+\frac{\left(\epsilon+\alpha_{4}\right)}{2} g_{\mu \nu} T_{\alpha \beta} T^{\alpha \beta}\right]+\left[\frac{3\left(\alpha_{6}+8 \alpha_{7}+\alpha_{4}\right)}{4\left(3+\alpha_{1}\right)\left(1+\epsilon \alpha_{1}\right)^{2}}\right] g_{\mu \nu} \sigma T .
\end{aligned}
$$

All these $4 D$ quantities have to be evaluated in the limit $y \rightarrow 0^{+}$. They give a working definition of the fundamental quantities $\Lambda_{4}$ and $G$ and contain higher-dimensional modifications to general relativity. As expected, switching off the effects of Lorentz violations $\left(\alpha_{i}=0\right)$ in these equations results in expressions one usually obtains in the brane-worlds models.

\section{Cosmological implications}

Assuming a perfect fluid configuration on the brane, the energy-momentum tensor is written as

$$
T_{\mu \nu}=(\rho+p) u_{\mu} u_{\nu}-p g_{\mu \nu}
$$

where $\mathbf{u}, \rho$ and $p$ are the unit velocity, energy density and pressure of the matter fluid respectively. We will also assume a linear isothermal equation of state for the fluid

$$
p=(\gamma-1) \rho, \quad 1 \leq \gamma \leq 2 .
$$

The weak energy condition [38] imposes the restriction $\rho \geq 0$. Now, it is useful to decompose ${ }^{(5)} E_{\mu \nu}$ with respect to any time-like observers $\mathbf{u}\left(u^{\mu} u_{\mu}=1\right)$ into a scalar part, $U$, a vector part, $Q_{\mu}$, and a tensorial part $P_{\mu \nu}[39]$, that is

$$
{ }^{(5)} E_{\mu \nu}=-\left(\frac{k_{5}}{k_{4}}\right)^{4}\left[\left(u_{\mu} u_{\nu}+\frac{1}{3} h_{\mu \nu}\right) U+2 u_{(\mu} Q_{\nu)}+P_{\mu \nu}\right],
$$

where the following properties hold

$$
Q_{\mu} u^{\mu}=0, \quad P_{\mu \nu}=P_{\nu \mu}, \quad P_{\mu}^{\mu}=0, \quad P_{\mu \nu} u^{\nu}=0 .
$$

Here, $Q_{\mu}$ is a spatial vector and $P_{\mu \nu}$ is a spatial, symmetric and trace-free tensor. The scalar term has the same form as the energy-momentum tensor of a radiation perfect fluid and for this reason $U$ is called the dark energy density [39]. From the modified Einstein equations (39) and equations (35) and (37) we may obtain a constraint on ${ }^{(5)} E_{\mu \nu}$ as has been done in [40].

In this paper we deal with non-tilted homogeneous cosmological models on the brane, i.e. we are assuming that the fluid velocity is orthogonal to the hypersurfaces of homogeneity. Also, we may 
consider $\phi\left(x^{\alpha}, y\right)=\phi(t)$. It is then convenient to make the splitting (45) with respect to the fluid velocity. In particular, we will consider FLRW models with the metric tensor given by

$$
d S^{2}=d t^{2}-a(t)^{2}\left[d r^{2}+\mathcal{Q}_{k}^{2}(r)\left(d \theta^{2}+\sin ^{2} \theta d \varphi^{2}\right)\right],
$$

where $\mathcal{Q}_{k}(r)=\sin (r), r, \sinh (r)$ corresponds to $k=1,0,-1$ respectively and $a(t)$ is the scale factor, with the fluid velocity given by $\mathbf{u}=\frac{\partial}{\partial t}$. Taking into account these assumptions and the effective Einstein's equations (39), the consequences of having a FLRW universe on the brane are

$$
Q_{\mu}=P_{\mu \nu}=0, \quad U=U(t) .
$$

The brane evolution can now be studied by determining the generalized Friedmann and Raychaudhuri equations in the presence of the vector field in the bulk. These follow from the Gauss-Codazzi equations and the time-like part of the trace of the Ricci identities applied to the time-like congruence $u_{\mu}$. Defining the parameters

$$
\begin{aligned}
\alpha_{8} & =\left(1+3(\gamma-1)^{2}\right)\left(2 \alpha_{4}-\alpha_{1}\right)+(4-3 \gamma)^{2}\left(\alpha_{6}+4 \alpha_{7}\right) \\
\alpha_{9} & =(4-3 \gamma)\left(\alpha_{6}+8 \alpha_{7}+\alpha_{4}\right) \\
\alpha_{10} & =\frac{\left(1+3(\gamma-1)^{2}\right) \alpha_{4}}{2}+(4-3 \gamma)^{2} \alpha_{7}+(4-3 \gamma) \alpha_{6}-\alpha_{1}
\end{aligned}
$$

these equations can be written as

$$
\begin{aligned}
H^{2} & =\frac{1}{3} \Lambda_{4}+\frac{1}{3}\left[1+\frac{\alpha_{9}}{\left(-\frac{2 \epsilon}{3}-2 \alpha_{1}+4 \alpha_{6}\right)}\right] k_{4}^{2} \rho+\frac{1}{3}\left[\frac{-\frac{\epsilon}{3}+\alpha_{10}}{\left(-\frac{2 \epsilon}{3}-2 \alpha_{1}+4 \alpha_{6}\right) \sigma}\right] k_{4}^{2} \rho^{2}+\left(\frac{k_{5}}{k_{4}}\right)^{4}\left(\frac{\epsilon+\alpha_{1}}{3+\alpha_{1}}\right) U(t) \\
& +\left[\frac{\alpha_{1}\left(\alpha_{5}-\frac{3}{2}\right)+3 \alpha_{5}}{3\left(3+\alpha_{1}\right)}\right] \frac{\ddot{\phi}}{\phi}+\left[\frac{\alpha_{5}\left(3-\alpha_{1}\right)}{6\left(3+\alpha_{1}\right)}\right]\left(\frac{\dot{\phi}}{\phi}\right)^{2}-\frac{k}{a(t)^{2}}
\end{aligned}
$$

and

$$
\begin{aligned}
\dot{H} & =-\frac{\gamma}{2} k_{4}^{2} \rho+\left[\frac{\left(2 \epsilon \gamma+\alpha_{8}-4 \alpha_{10}\right)}{6\left(-\frac{2 \epsilon}{3}-2 \alpha_{1}+4 \alpha_{6}\right) \sigma}\right] k_{4}^{2} \rho^{2}-\left(\frac{k_{5}}{k_{4}}\right)^{4}\left[\frac{2\left(\epsilon+\alpha_{1}\right)}{3+\alpha_{1}}\right] U(t) \\
& +\left[\frac{\alpha_{1}}{\left(3+\alpha_{1}\right)}\right] \frac{\ddot{\phi}}{\phi}-\left[\frac{2 \alpha_{5}}{\left(3+\alpha_{1}\right)}\right]\left(\frac{\dot{\phi}}{\phi}\right)^{2}+\frac{k}{a(t)^{2}},
\end{aligned}
$$

allowing us to examine the evolution of the brane without using any particular solution of the fivedimensional field equations. It is worth noting again that if the effects of Lorentz violations are ignored $\left(\alpha_{i}=0\right)$, the above equations reduce to the usual Friedmann and Raychaudhuri equations in the brane-world scenarios [40].

\subsection{Variable vacuum energy}

In the above equations $G$ and $\Lambda_{4}$ are usually assumed to be truly constants. In this section we show how equations (50) and (51) should be modified as to incorporate the variation of these fundamental physical "constants," matching observational predictions. We can make a simplification without loss of generality by setting $\beta_{1}=2 \beta_{2}$ and $\beta_{3}=\frac{-5}{2} \beta_{2}$. Also noting that $\alpha_{1}<-3$, we obtain $\beta_{2}>3$. From (35), (36) and (37) it follows that

$$
\sigma_{, \nu}+T_{\nu ; \mu}^{\mu}=-\alpha_{2}(4 \sigma+T)_{, \nu}
$$

where $\alpha_{2}=\frac{\epsilon \beta_{2}}{3-7 \epsilon \beta_{2}}$. For the perfect fluid, equation (43), this is equivalent to

$$
\left(1+\alpha_{2}(4-3 \gamma)\right) \dot{\rho}+(\rho+p) \Theta=-\left(1+4 \alpha_{2}\right) \dot{\sigma},
$$


and

$$
(\rho+p) a_{\nu}+\left[(\gamma-1)-\alpha_{2}(4-3 \gamma)\right] \rho_{, \lambda} h_{\nu}^{\lambda}=\left(1+4 \alpha_{2}\right) \sigma_{, \lambda} h_{\nu}^{\lambda}
$$

where $\Theta=u_{; \mu}^{\mu}$ is the usual expression for expansion, $a_{\nu}=u_{\nu ; \lambda} u^{\lambda}$ is the acceleration and $h_{\mu \nu}=$ $u_{\mu} u_{\nu}-g_{\mu \nu}$ is the projection onto the spatial surfaces orthogonal to $u_{\mu}$. In homogeneous cosmological models, equation (54) becomes redundant and only equation (53) is relevant. For the case of constant vacuum energy $\sigma$ and equation of state $p=(\gamma-1) \rho$, it yields the familiar relation between the matter energy density and expansion factor $a$

$$
\rho=\rho_{0}\left(\frac{a_{0}}{a}\right)^{\frac{3 \gamma}{1+\alpha_{2}(4-3 \gamma)}} .
$$

For the case where the vacuum energy is not constant we need some additional assumption. For example, $\sigma$ may be a function of the scale factor, $\sigma=\sigma(a)$. However, we have so far no theoretical observational arguments for the evolution of $\sigma$ in time.

\subsection{Time evolution of the universe}

The time variation of $G$ is usually written as $\left(\frac{\dot{G}}{G}\right)=g H$, where $g$ is a dimensionless parameter. Nucleosynthesis and the abundance of various elements are used to put constraints on $g$. The present observational upper bound is $|g| \leq 0.1[41,42]$. In what follows we assume that $g$ is constant. Since $G \sim \sigma$ and $H=\frac{\dot{a}}{a}$ we have $\sigma(a)=f_{0} a^{g}$, where $f_{0}$ is a constant of integration. From equation (53) we thus find

$$
\left[1+\alpha_{2}(4-3 \gamma)\right] \dot{\rho}+3 \gamma \rho \frac{\dot{a}}{a}=-\left(1+4 \alpha_{2}\right) f_{0} g a^{(g-1)} \dot{a} .
$$

First, we consider the case where $\rho$ can be expressed in a way similar to (55), i.e. as a power function of $a$. We therefore find

$$
g=\frac{-3 \gamma D}{D+f_{0}+\alpha_{2}\left(4 D-3 \gamma D+4 f_{0}\right)}
$$

and

$$
\rho=D a^{-\left[\frac{3 \gamma D}{D+f_{0}+\alpha_{2}\left(4 D-3 \gamma D+4 f_{0}\right)}\right]},
$$

where $D$ is a positive constant. In order to simplify the notation we set $f_{0}=F_{0} D$ and

$$
\frac{\gamma}{1+F_{0}+\alpha_{2}\left(4-3 \gamma+4 F_{0}\right)}=\zeta+1
$$

which gives

$$
\zeta=\frac{\gamma-F_{0}-1-\alpha_{2}\left(4-3 \gamma+4 F_{0}\right)}{1+F_{0}+\alpha_{2}\left(4-3 \gamma+4 F_{0}\right)} .
$$

With this notation and substituting $\alpha_{2}$, we have

$$
\rho=\frac{D}{a^{3(\zeta+1)}} \quad \text { and } \quad \sigma=\left[\frac{\left(3-7 \epsilon \beta_{2}\right) D \gamma-3 D(\zeta+1)\left(1-\epsilon \beta_{2}-\epsilon \gamma \beta_{2}\right)}{3(\zeta+1)\left(1-\epsilon \beta_{2}\right)}\right] a^{-3(\zeta+1)} .
$$

Note that one may take $D=\rho_{0} a_{0}^{3(\zeta+1)}$ and that $F_{0} \neq 0\left(\zeta \neq \frac{\gamma-\left(1+\alpha_{2}(4-3 \gamma)\right)}{\left(1+\alpha_{2}(4-3 \gamma)\right)}\right)$, otherwise $G=0$. Here, equations (40) and (41) can be written as follows

$$
\begin{aligned}
\Lambda_{4} & =\frac{k_{5}^{2}}{2} \Lambda_{5}+\left[\frac{-\frac{3}{4} \epsilon+\frac{3}{2} \beta_{2}-\frac{1}{2} \beta_{2}^{2}-\frac{65}{6} \epsilon \beta_{2}+\frac{29}{3} \epsilon \beta_{2}^{2}+\frac{17}{36} \epsilon \beta_{2}^{3}}{\left(3-\beta_{2}\right)\left(\epsilon-\beta_{2}\right)^{2}\left(3-7 \epsilon \beta_{2}\right)}\right] \\
& \times\left[\frac{\left(3-7 \epsilon \beta_{2}\right) D \gamma-3 D(\zeta+1)\left(1-\epsilon \beta_{2}-\epsilon \gamma \beta_{2}\right)}{3(\zeta+1)\left(1-\epsilon \beta_{2}\right)}\right]^{2} k_{5}^{4} a^{-6(\zeta+1)}, \\
8 \pi G & =\left[\frac{-3 \epsilon k_{5}^{4}}{2\left(3-\beta_{2}\right)\left(3-7 \epsilon \beta_{2}\right)}\right]\left[\frac{\left(3-7 \epsilon \beta_{2}\right) D \gamma-3 D(\zeta+1)\left(1-\epsilon \beta_{2}-\epsilon \gamma \beta_{2}\right)}{3(\zeta+1)\left(1-\epsilon \beta_{2}\right)}\right] a^{-3(\zeta+1)} .
\end{aligned}
$$


We also have

$$
\frac{\dot{G}}{G}=-3(\zeta+1) H
$$

Before going any further, we should be aware of the observational bounds on $\zeta$. The lower bound comes from the obvious requirement $\frac{d \rho}{d a}<0$, while the upper bound comes from the observation that $|g| \leq 0.1$. Thus,

$$
-1<\zeta \leq-0.966, \quad \zeta=-0.983 \pm 0.016 .
$$

We can see that in general $\zeta$ is related to $q$ and $\Omega_{\rho}$, the deceleration and density parameters respectively. Another point of interest is that if one assumes a constant bulk cosmological constant, equations (40) and (41) imply $\dot{\Lambda}_{4} \sim G \dot{G}$, a relation frequently encountered in brane theories.

The next step would be to obtain the evolution equation for $a$. In the standard cosmological models, we can set $\phi=\phi_{0}\left(\frac{a}{a_{0}}\right)^{r}$. The physical condition $\tau_{0}^{0}=(\sigma+\rho)>0$ then puts a lower limit on $r$, namely $r>1$ [36]. Now, substituting equations (60) and (61) into

$$
\begin{aligned}
\left(2-\frac{2}{3} \epsilon r^{2} \beta_{2}\right) H^{2}+\left(1-\frac{4}{3} \epsilon r \beta_{2}\right) \dot{H} & =\frac{2}{3} \Lambda_{4}+\frac{2}{3}\left[\frac{4-3 \gamma}{4}+\frac{\alpha_{9}}{\left(-\frac{2 \epsilon}{3}-2 \alpha_{1}+4 \alpha_{6}\right)}\right] k_{4}^{2} \rho \\
& +\left[\frac{-\frac{2}{3}(2-3 \gamma) \epsilon+\alpha_{8}}{6\left(-\frac{2 \epsilon}{3}-2 \alpha_{1}+4 \alpha_{6}\right) \sigma}\right] k_{4}^{2} \rho^{2}-\frac{k}{a^{2}},
\end{aligned}
$$

which is obtained by combining equations (50) and (51), and introducing the quantities

$$
\begin{aligned}
e^{x} & =a^{3(\zeta+1)}, \\
s_{1} & =\left[\frac{6 D^{2}(\zeta+1)}{\left(3-4 \epsilon r \beta_{2}\right)}\right]\left[\frac{-\frac{3}{4} \epsilon+\frac{3}{2} \beta_{2}-\frac{1}{2} \beta_{2}^{2}-\frac{65}{6} \epsilon \beta_{2}+\frac{29}{3} \epsilon \beta_{2}^{2}+\frac{17}{36} \epsilon \beta_{2}^{3}}{\left(3-\beta_{2}\right)\left(\epsilon-\beta_{2}\right)^{2}\left(3-7 \epsilon \beta_{2}\right)}\right] \\
& \times\left[\frac{\left(3-7 \epsilon \beta_{2}\right) \gamma-3(\zeta+1)\left(1-\epsilon \beta_{2}-\epsilon \gamma \beta_{2}\right)}{3(\zeta+1)\left(1-\epsilon \beta_{2}\right)}\right]^{2} k_{5}^{4}, \\
s_{2} & =\left[\frac{(4-3 \gamma) D^{2}}{3-4 \epsilon r \beta_{2}}\right]\left[\frac{-\frac{3}{4} \epsilon+\frac{3}{2} \beta_{2}-\frac{1}{2} \beta_{2}^{2}-\frac{65}{6} \epsilon \beta_{2}+\frac{29}{3} \epsilon \beta_{2}^{2}+\frac{17}{36} \epsilon \beta_{2}^{3}}{\left(3-\beta_{2}\right)\left(\epsilon-\beta_{2}\right)^{2}\left(3-7 \epsilon \beta_{2}\right)}\right] \\
& \times\left[\frac{\left(3-7 \epsilon \beta_{2}\right) \gamma-3(\zeta+1)\left(1-\epsilon \beta_{2}-\epsilon \gamma \beta_{2}\right)}{\left(1-\epsilon \beta_{2}\right)}\right] k_{5}^{4}, \\
s_{3} & =\left[\frac{D^{2}(\zeta+1)(4-3 \gamma)^{2}}{3-4 \epsilon r \beta_{2}}\right]\left[\frac{3+9(\gamma-1)^{2}}{8\left(\epsilon-\beta_{2}\right)(4-3 \gamma)^{2}}+\frac{9\left(\epsilon-\beta_{2}\right)\left(1-3 \epsilon \beta_{2}\right)+2 \epsilon \beta_{2}^{2}\left(1+\beta_{2}\right)}{8\left(3-7 \epsilon \beta_{2}\right)\left(3-\beta_{2}\right)\left(1-\epsilon \beta_{2}\right)^{2}}\right. \\
& \left.+\frac{\beta_{2}^{2}}{12\left(3-\beta_{2}\right)\left(1-\epsilon \beta_{2}\right)^{2}}+\frac{\left(-6+\beta_{2}\right)}{8\left(3-\beta_{2}\right)\left(\epsilon-\beta_{2}\right)}+\frac{\beta_{2}}{24\left(1-\epsilon \beta_{2}\right)^{2}}\right] k_{5}^{4},
\end{aligned}
$$

with

$$
s=s_{1}+s_{2}+s_{3},
$$

we obtain the following equation

$$
\ddot{x}+\left[\frac{6-2 \epsilon r^{2} \beta_{2}}{3(\zeta+1)\left(3-4 \epsilon r \beta_{2}\right)}\right] \dot{x}^{2}=\left[\frac{3(\zeta+1)}{3-4 \epsilon r \beta_{2}}\right] k_{5}^{2} \Lambda_{5}+s e^{-2 x}-\left[\frac{9(\zeta+1)}{3-4 \epsilon r \beta_{2}}\right] k e^{\frac{-2 x}{3(\zeta+1)}} .
$$

Let us concentrate on the evolution of the universe at the present epoch. In this case the exponential terms become small and could be ignored. As it can be seen, the coefficient of $\dot{x}^{2}$ is always positive. Therefore, the evolution of the universe not only depends on the bulk cosmological constant being negative, zero or positive but also depends on the extra dimension being timelike or spacelike. We study these cases separately in what follows. 


\subsubsection{Anti-de Sitter bulk, $\epsilon>0$}

This case is important since it corresponds to the brane-world scenarios where our universe is identified with a singular hypersurface embedded in an $\mathrm{AdS}_{5}$ bulk. The evolution of the scale factor is given by

$$
a=a_{0}\left(\frac{\cosh \theta}{\cosh \theta_{0}}\right)^{\frac{1}{3(\zeta+1) E_{1}}}
$$

where

$$
\begin{gathered}
\theta=\sqrt{E_{1} E_{2}} t^{\prime}+\tanh ^{-1}\left(3(\zeta+1) H_{0} \sqrt{\frac{E_{1}}{E_{2}}}\right), \quad t^{\prime}=t-t_{0}, \\
E_{1}=\left[\frac{6-2 \epsilon r^{2} \beta_{2}}{3(\zeta+1)\left(3-4 \epsilon r \beta_{2}\right)}\right], \quad E_{2}=\left[\frac{3(\zeta+1)}{3-4 \epsilon r \beta_{2}}\right] k_{5}^{2} \Lambda_{5} .
\end{gathered}
$$

The zero subscript denotes the measurement of the quantity at the present epoch and $H$ is the Hubble parameter. Simply, using equations (60) and (61), one can obtain the time varying forms of $\Lambda_{4}$ and $G$. The deceleration parameter $q$ defined by $q=\frac{-\ddot{a} a}{\dot{a}^{2}}$ becomes

$$
q=-\left[\frac{3(\zeta+1) E_{1}\left(1-\tanh ^{2} \theta\right)+\tanh ^{2} \theta}{\tanh ^{2} \theta}\right],
$$

which is always negative. Therefore, according to the approximation made to solve equation (66), one has late time accelerated expansion of the universe. We note that for a spacelike extra dimension the time-evolution of the brane in a $\mathrm{dS}_{5}$ bulk is again given by (67).

We note that a non-vanishing cosmological constant in the bulk induces a natural time scale in $4 D$, defined as $\tau_{s}=\sqrt{3 / \tilde{\Lambda}}$ where $\tilde{\Lambda} \equiv \frac{k_{5}^{2} \Lambda_{5}}{2\left(1-\frac{\epsilon}{3} r^{2} \beta_{2}\right)}$ [36]. The influence of Lorentz violation in the value of $\tilde{\Lambda}$ is embodied in the factor $\beta_{2}$ and the signature of the extra dimension. In the absence of Lorentz violation, $\beta_{2}=0$ and one gets the usual expression. The late time behavior of solutions (67) becomes identical to the one in the de Sitter solution

$$
a\left(t^{\prime}\right) \sim \exp \left(\frac{t^{\prime}}{\tau_{s}}\right)
$$

as $t^{\prime} \gg \tau_{s}$. This exponential behavior is not a consequence of the false-vacuum equation of state $p=-\rho$ as in inflation since $\gamma \neq 0$ here. The reason for this is that the assumption $\frac{\dot{G}}{G}=g H$ is equivalent to the requirement that $\rho$ and $\sigma$ form a combined fluid with energy density $\tilde{\rho}=\rho+\sigma$ and pressure $\tilde{p}=\zeta \tilde{\rho}$. Then, the observational constraint $-1<\zeta \leq-0.966$ implies that the combined fluid behaves nearly like a cosmological constant, which dominates at late times and thus producing inflation.

\subsubsection{Bulk with $\Lambda_{5}=0$}

In this case, the expansion factor is given by

$$
a=a_{0}\left[3(\zeta+1) H_{0} E_{1} t^{\prime}+1\right]^{\frac{1}{3(\zeta+1) E_{1}}} .
$$

One then obtains the deceleration parameter $q$ as follows

$$
q=-\left[\frac{-3+2 \epsilon r(r-2) \beta_{2}}{3-4 \epsilon r \beta_{2}}\right] .
$$

To be in agreement with observations, the deceleration parameter $q$ must be negative. This leads to the following condition

$$
r(r-2)<\frac{3 \epsilon}{2 \beta_{2}}
$$

making the functional form of $\phi$ somewhat restricted. 


\subsection{3 de Sitter bulk, $\epsilon>0$}

Although in brane-world theories our universe is embedded in a higher-dimensional space with negative cosmological constant, the solutions to the evolution equation depends analytically on the sign of $E_{2}$, allowing us to consider the negative values of $E_{2}$ which would include a positive cosmological constant, provided the extra dimension is taken to be timelike. The evolution of the scale factor is given by

$$
a=a_{0}\left(\frac{\cos \varphi}{\cos \varphi_{0}}\right)^{\frac{1}{3(\zeta+1) E_{1}}}
$$

where

$$
\varphi=-\sqrt{-E_{1} E_{2}} t^{\prime}+\tan ^{-1}\left(3(\zeta+1) H_{0} \sqrt{\frac{-E_{1}}{E_{2}}}\right),
$$

and the deceleration parameter is found to be

$$
q=-\left[\frac{3(\zeta+1) E_{1}\left(1+\tan ^{2} \varphi\right)+\tan ^{2} \varphi}{\tan ^{2} \varphi}\right] .
$$

In this case, $q$ is always negative, leading to an accelerated expanding universe. Note that for $\epsilon<0$, the brane evolution in an $\mathrm{AdS}_{5}$ bulk is again given by (75).

\section{Conclusions}

In this paper we have studied a brane-world scenario whereupon the idea of Lorentz violation, introduced by specifying a preferred frame through the introduction of a dynamical vector field normal to our brane has been considered. Such a normal vector was, however, assumed to be decoupled from the matter fields since such fields were assumed to be confined to the brane. The Einstein field equations were obtained on the brane using the SMS formalism, modified by the additional vector field. To study the cosmological implication of our brane-world, Lorentz violating model, we focused attention on a Robertson-Walker background. The ensuing Friedmann and Raychaudhuri equations were analyzed in various cases where the bulk cosmological constant was negative or positive together with the signature of the extra dimension being timelike or spacelike. We found that an accelerated expanding universe results in all these cases. For a zero bulk cosmological constant, acceleration is possible only if a certain constraint on $r$ is satisfied. Also, it was shown that local Lorentz violation in the bulk allows for the construction of models in which the vacuum energy $\sigma$, the gravitational coupling $G$ and the cosmological term $\Lambda_{4}$ are variable.

\section{References}

[1] For a couple of recent reviews, see for example Mattingly D, Preprint gr-qc/0502097; Vucetich H., Preprint gr-qc/0502093; Jacobson T, Liberate S and Mattingly D, Preprint astro-ph/0505267.

[2] Talk presented at CPT01; the Second Meeting on CPT and Lorentz Symmetry, Bloomington, Indiana, 15-18 Aug. 2001.

[3] Connes A, Douglas M R and Schwarz A 1998 JHEP 02003 (Preprint hep-th/9711162).

[4] Carroll S M, Harvey J A, Kostelecky V A, Lane C D and Okamoto T 2001 Phys. Rev. Lett. 87 141601 (Preprint hep-th/0105082).

[5] Zatsepin G T and Kuzmin V A 1966 Prima Zh. Eksp. Teor. Fiz 4114.

[6] Chisholm J R and Kolb E W 2004 Phys. Rev. D 69085001 (Preprint hep-ph/0306288). 
[7] Dubovsky S L and Tinyakov P G 2002 Astropart. Phys. 1889 (Preprint astro-ph/0106472).

[8] Coleman S R and Glashow S L 1999 Phys. Rev. D 59116008 (Preprint hep-ph/9812418).

[9] Vankov H and Stanev T 2002 Phys. Lett. B 538251 (Preprint astro-ph/0202388).

[10] Zee A 1982 Phys. Rev. D 251864.

[11] Aloisio R, Blasi P, Ghia P L and Grillo A F 2000 Phys. Rev. D 62053010 (Preprint astroph/0001258).

[12] Bertolami O and Carvalho C S 2000 Phys. Rev. D 61103002 (Preprint gr-qc/9912117).

[13] Alfaro J and Palma G 2003 Phys. Rev. D 67083003 (Preprint hep-th/0208193).

[14] Coleman S R and Glashow S L 1997 Phys. Lett. B 405249 (Preprint hep-ph/9703240).

[15] Kostelecky V A and Samuel S 1989 Phys. Rev. D 401886.

[16] Bekenstein J D 2004 (Preprint astro-ph/0403694).

[17] Nordtvedt K J and Will C M 1972 Astrophys. J. 177775.

[18] Ford L H 1989 Phys. Rev. D 40967.

[19] Dolgov A D 1985 JETP Lett. 41345.

[20] Kiselev V V 2004 Class. Quantum Grav. 213323 (Preprint gr-qc/0402095).

[21] Colladay D and Kostelecky V A 1998 Phys. Rev. D 58116002 (Preprint hep-ph/9809521).

[22] Carroll S M, Field G B and Jackiw R 1990 Phys. Rev. D 411231.

[23] Coleman S R and Glashow S L 1999 Phys. Rev. D 59116008 (Preprint hep-ph/9812418).

[24] Aloisio R, Blasi P, Ghia P L and Grillo A F 2000 Phys. Rev. D 62053010 (Preprint astroph/0001258).

[25] Csaki C, Erlich J and Grojean C 2001 Nucl. Phys. B 604312 (Preprint hep-th/0012143).

[26] Burgess C P, Cline J, Filotas E, Matias J and Moore G D 2002 JHEP 0203043 (Preprint hep-ph/0201082).

[27] Stoica H 2002 JHEP 0207060 (Preprint hep-th/0112020).

[28] Libanov M V and Rubakov V A 2005 JCAP 0509005 (preprint astro-ph/0504249).

[29] Jacobson T and Mattingly D 2001 Phys. Rev. D 64024028.

[30] Kostelecky V A 2004 Phys. Rev. D 69105009 (Preprint hep-th/0312310).

[31] Shiromizu T, Maeda K and Sasaki M 2000 Phys. Rev. D 6202412 (Preprint gr-qc/9910076).

[32] Carroll S M, Lim E A 2004 Phys. Rev. D 70123525 (Preprint hep-th/0407149).

[33] Lim E A 2005 Phys.Rev. D 71063504 (Preprint astro-ph/0407437).

[34] Eling C, Jacobson T, Mattingly D to appear in the Deserfest proceedings (World Scientific) (Preprint gr-qc/0410001).

[35] Jacobson T and Mattingly D 2004 (Preprintgr-qc/0402005). 
[36] Ponce de Leon J 2003 Class. Quantum Grav. 205321 (Preprintgr-qc/0305041), Ponce de Leon J 2002 Mod. Phys. Lett. A 172425 (Preprintgr-qc/0207001).

[37] Ponce de Leon J 2002 Int. J. Mod. Phys. D 111355 (Preprint gr-qc/0105120).

[38] Hawking S W and Ellis G F R The large scale structure of space-time (Cambridge University Press, Cambridge, 1973).

[39] Maartens R 2000 Phys. Rev. D 62084023.

[40] Campos A, Sopuerta C F 2001 Phys. Rev. D 64104011.

[41] Uzan J P 2003 Rev. Mod. Phys. 75403 (Preprint hep-ph/0205340).

[42] Bronnikov K A, Melnikov V N and Novello M 2002 Gravitation and Cosmology Suppl. 218 (Preprint gr-qc/0208028). 